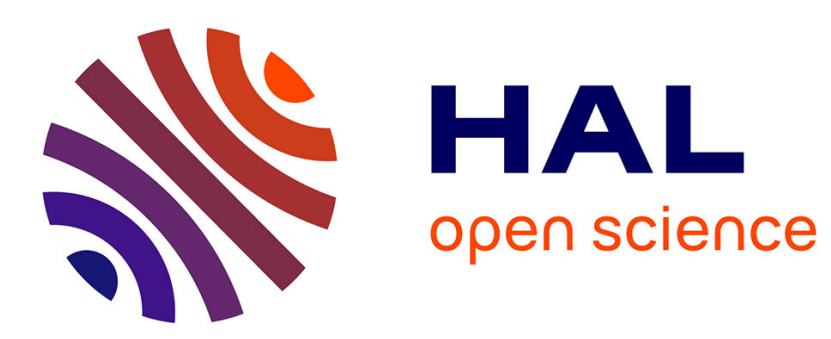

\title{
Reduced Order LQG Control Design for Infinite Dimensional Port-Hamiltonian Systems
}

Yongxin Wu, Boussad Hamroun, Yann Le Gorrec, Bernhard Maschke

\section{To cite this version:}

Yongxin Wu, Boussad Hamroun, Yann Le Gorrec, Bernhard Maschke. Reduced Order LQG Control Design for Infinite Dimensional Port-Hamiltonian Systems. IEEE Transactions on Automatic Control, 2021, 66 (2), pp.865 - 871. 10.1109/TAC.2020.2997373 . hal-03132036

\section{HAL Id: hal-03132036 https://hal.science/hal-03132036}

Submitted on 19 Feb 2021

HAL is a multi-disciplinary open access archive for the deposit and dissemination of scientific research documents, whether they are published or not. The documents may come from teaching and research institutions in France or abroad, or from public or private research centers.
L'archive ouverte pluridisciplinaire HAL, est destinée au dépôt et à la diffusion de documents scientifiques de niveau recherche, publiés ou non, émanant des établissements d'enseignement et de recherche français ou étrangers, des laboratoires publics ou privés. 


\title{
Reduced Order LQG Control Design for Infinite Dimensional Port Hamiltonian Systems
}

\author{
Yongxin Wu, Member, IEEE, Boussad Hamroun, Member, IEEE, Yann Le Gorrec, Senior Member, IEEE, \\ Bernhard Maschke, Member, IEEE
}

\begin{abstract}
This paper proposes a method that combines LQG control design and structure preserving model reduction for the reduced order control of Infinite Dimensional Port Hamiltonian Systems (IDPHS). For that purpose the weighting operators used in LQG control design are chosen such that the resulting dynamic controller is passive and the closed-loop system equivalent to control by interconnection. The method of Petrov-Galerkin is then used to approximate the balanced realization of the IDPHS by a finite dimensional port Hamiltonian system and to provide the associated reduced order LQG controller. The main advantages of the proposed method are that, first, both control and reduction are driven by closed-loop performances and that, second, due to the passivity properties of the controller the closed-loop stability is guaranteed when the finite dimensional controller is applied to the infinite dimensional system.
\end{abstract}

Index Terms - Infinite dimensional systems, port Hamiltonian systems, LQG method, model reduction, controller reduction.

\section{INTRODUCTION}

In this paper we consider the reduced order control design for infinite dimensional port Hamiltonian systems modelled by linear PDEs. The aim is to design a reduced order controller able to achieve a given set of performances on the reduced order system while guaranteeing the closed-loop stability when applied to the infinite dimensional system.

There exists an extensive literature on reduced order control design for infinite dimensional systems. First, the early lumping approaches consist in reducing the open loop system and designing a controller by using a classical control design method. Even when port Hamiltonian formulations and passivity based control are concerned, these approaches fail when weakly damped or undamped systems (hyperbolic systems) are considered. In this case the state variables of the open loop system have the same weight and the reduction techniques fail in providing suitable models for control design purposes. Furthermore neglected high frequency modes often become unstable when the loop is closed. This phenomenon is well known as spillover effect [1]. Second, the late lumping approach consists in designing the control law on the infinite dimensional system or on a high order approximation of the

Yongxin Wu and Yann Le Gorrec are with FEMTO-ST, UMR CNRS 6174, AS2M Department, Université de Bourgogne Franche-Comté, Universit de Franche-Comté, ENSMM, 24 rue Savary, F-25000 Besanon, France, e-mail: yongxin.wu@femto-st.fr.

Boussad Hamroun and Bernhard Maschke are with Université de Lyon, F-69622, Lyon, France; Université Claude Bernard Lyon 1, Villeurbanne; LAGEP, UMR5007, CNRS.

This work was supported by the ANR-DFG (French-German) project INFIDHEM (contract "ANR-16-CE92-0028") and the Bourgogne-Franchecomté Region ANER project (contract "2018Y-06145"). system and then in proceeding to the reduction of the controller. This closed-loop reduction/control design method has been developed using port Hamiltonian formulations for large scale finite dimensional systems in [2] but has been hardly considered in the infinite dimensional case. More precisely in [2] LQG control, balanced realization and structure preserving reduction are combined for the efficient design of reduced order controllers for large scale systems.

With the same idea in mind, this paper aims to propose a passive reduced order LQG control design method for infinite dimensional port Hamiltonian systems (IDPHS) with distributed control in the domain and no boundary energy exchange. The LQG method is used to develop a LQG based model reduction which takes into account the closed-loop considerations in [3], [4], [5] for infinite dimensional systems. The main drawback of such method is that it does not preserve the passivity properties of the system in open loop nor in closedloop because LQG control design is not a passive control design method in general. To get a passive reduced order LQG controller in this paper, we first derive the conditions under which a LQG controller is passive and equivalent to a Control by Interconnection [6]. From this LQG approach we define a balanced basis in which the LQG Hankel operator is nuclear with summable singular values. The reduction procedure then uses the Petrov-Galerkin approximation [7] preserving both the port Hamiltonian structure and the passivity of the system.

This paper is organized as follows. In Section II, we introduce a class of infinite dimensional passive systems that can be represented in the port Hamiltonian framework. In Section III we adapt the LQG method to these infinite dimensional port Hamiltonian systems such that the resulting controller is passive and equivalent to the control by interconnection. In Section IV we use this LQG formulation to derive a reduction scheme that preserves the port Hamiltonian structure and in consequence the passivity of the controller. The stability of the closed-loop system when the finite dimensional controller is applied to the infinite dimensional system is then proven. We illustrate and compare the full order and reduced order LQG controllers on the Timoshenko beam control example in Section V. The last Section gives some conclusions and perspectives.

\section{A CLASS OF INFINITE DIMENSIONAL PORT HAMILTONIAN SYSTEM}

We consider the class of linear infinite-dimensional dissipative systems defined as follows: 
Definition 1. A linear infinite-dimensional system of the form:

$$
\Sigma_{P H S}\left\{\begin{aligned}
\dot{x}(t) & =\mathcal{M} \mathcal{Q} x(t)+\mathcal{B} u(t) \\
y(t) & =\mathcal{B}^{*} \mathcal{Q} x(t)
\end{aligned}\right.
$$

is called a linear infinite-dimensional dissipative portHamiltonian system (IDPHS) if it satisfies

- $x(t) \in X, X$ is a Hilbert space with inner product $\langle\cdot, \cdot\rangle_{X}$ and norm $\|\cdot\|_{X}^{2}$;

- $\mathcal{M}: D(\mathcal{M}) \subset X$, the domain of the operator $\mathcal{M}$ is a densely definite maximal dissipative ( $m$-dissipative) linear operator;

- $\mathcal{Q}: X \mapsto X$ is a bounded linear operator that is selfadjoint $\left(\mathcal{Q}^{*}=\mathcal{Q}\right)$ and coercive $\left(\langle\mathcal{Q} h, h\rangle_{X} \geq \alpha\|h\|_{X}^{2}\right.$ $\forall h \in X$ with $\alpha>0$ );

- The input operator $\mathcal{B}: \mathbb{C}^{p} \mapsto X$ is bounded and $\{0\} \neq$ $\operatorname{Im}(\mathcal{B}) \subset X$.

- The inputs $u$ and outputs y have the same dimension.

The operator $\mathcal{M Q}$ is dissipative with respect to the inner product $\langle g, h\rangle_{\mathcal{Q}}=\langle g, \mathcal{Q} h\rangle_{X}, g, h \in X$. In addition, $\operatorname{Ran}(\lambda I-$ $\mathcal{M Q})=X$ is satisfied for some $\lambda \in \mathbb{C}_{0}$, because $\mathcal{M}$ is $m$ dissipative and $\mathcal{Q}$ is bijective. Hence $\mathcal{M Q}$ is $m$-dissipative and therefore generates a contraction $C_{0}$-semigroup [8, Thm. 1.2.3].

The total energy of the system is defined by the Hamiltonian (Energy storage equation) as

$$
H(x(t))=\frac{1}{2}\langle\mathcal{Q} x(t), x(t)\rangle_{X}
$$

Then the power balance equation of the system is given by

$$
\begin{aligned}
\frac{d}{d t} H(x(t)) & =\frac{1}{2}\langle\mathcal{Q} x(t), \dot{x}(t)\rangle_{X}+\frac{1}{2}\langle\mathcal{Q} \dot{x}(t), x(t)\rangle_{X} \\
& =\frac{1}{2}\langle\mathcal{Q} x(t), \dot{x}(t)\rangle_{X}+\frac{1}{2}\left\langle\dot{x}(t), \mathcal{Q}^{*} x(t)\right\rangle_{X}
\end{aligned}
$$

Due to the relation $\mathcal{Q}^{*}=\mathcal{Q}$, we have

$$
\begin{aligned}
\operatorname{Re}\langle\mathcal{Q} \dot{x}(t), x(t)\rangle_{X} & =\operatorname{Re}\langle\dot{x}(t), \mathcal{Q} x(t)\rangle_{X} \\
& =\operatorname{Re}\langle\mathcal{Q} x(t), \dot{x}(t)\rangle_{X} .
\end{aligned}
$$

Thus by taking equation (1) into account, one get

$$
\begin{aligned}
\frac{d}{d t} H(x(t)) & =\operatorname{Re}\langle\mathcal{Q} x(t), \dot{x}(t)\rangle_{X} \\
& =\operatorname{Re}\langle\mathcal{Q} x(t), \mathcal{M} \mathcal{Q} x(t)+\mathcal{B} u(t)\rangle_{X}
\end{aligned}
$$

and $\langle\mathcal{Q} x(t), \mathcal{B} u(t)\rangle_{X}=\left\langle\mathcal{B}^{*} \mathcal{Q} x(t), u(t)\right\rangle_{\mathbb{C}_{p}}=y^{T}(t) u(t)$. Considering the $m$-dissipativity of $\mathcal{M}^{p}$ we have that $\operatorname{Re}\langle\mathcal{Q} x(t), \mathcal{M} \mathcal{Q} x(t)\rangle_{X} \leq 0$ and then from the above equations, we can get

$$
\frac{d}{d t} H(x(t)) \leq y^{T}(t) u(t)
$$

From the physical point view, we can interpret that the variation of total energy is lower than or equal to the power fed into the system. If we consider that $H$ admits a lower bound, due to this property, the infinite dimensional system (1) is passive [9]. Therefore, the system does not contain any internal sources.

Assumption 2. Through out this paper, we suppose the domain of the operator $\mathcal{M}$ is equal to the domain of $\mathcal{M}^{*}$, i.e.,

$$
D\left(\mathcal{M}^{*}\right)=D(\mathcal{M})
$$

By using Assumption 2, the system (1) can be written as:

$$
\left\{\begin{aligned}
\dot{x}(t) & =(\mathcal{J}-\mathcal{R}) \mathcal{Q} x(t)+\mathcal{B} u(t) \\
y(t) & =\mathcal{B}^{*} \mathcal{Q} x(t)
\end{aligned}\right.
$$

where

$$
\mathcal{J}=\frac{1}{2}\left(\mathcal{M}-\mathcal{M}^{*}\right) \quad \text { and } \quad \mathcal{R}=-\frac{1}{2}\left(\mathcal{M}+\mathcal{M}^{*}\right)
$$

with $D(\mathcal{J})=D(\mathcal{R})=D(\mathcal{M}) \subset X$. Hence the system (1) can be regarded as an infinite dimensional port Hamiltonian system (IDPHS) defined in [10]. Here the operator $\mathcal{J}=-\mathcal{J}^{*}$ is a skew-adjoint differential operator which present the energy exchange in the domain, and the operator $\mathcal{R}=\mathcal{R}^{*}$ is a semi positive definite self-adjoint differential operator which represents the energy dissipation in the domain.

It should be noted that the input mapping $\mathcal{B} \in \mathcal{L}\left(\mathbb{C}^{p} ; X\right)$ is bounded and $\{0\} \neq \operatorname{Im} \mathcal{B} \subset X$. The considered systems do not exchange energy through their boundary and that in domain control is considered.

Note that the conjugated inputs and outputs whereas fintedimensional correspond to distributed control and sensing, contrary to the boundary controlled PHS considered in [10], [11]. This class of systems is not considered in this paper, it shall be studied in future works.

Example 3. Clamped-free Timoshenko beam. We consider the camped-free beam with internal damping and distributed control depicted in Figure 1. It can be formulated as a 1-D port Hamiltonian system [12], [13] as follows (with $z \in[0,1]$ ):

$$
\left\{\begin{aligned}
\dot{x} & =\mathcal{M} \mathcal{Q} x+\mathcal{B} u(t) \\
y & =\mathcal{B}^{*} \mathcal{Q} x
\end{aligned}\right.
$$

where $x^{T}=\left[x_{1}, x_{2}, x_{3}, x_{4}\right]^{T}$ and:

$\mathcal{M}=\left[\begin{array}{cccc}0 & \frac{\partial}{\partial z} & 0 & -1 \\ \frac{\partial}{\partial z} & -R_{p} & 0 & 0 \\ 0 & 0 & 0 & \frac{\partial}{\partial z} \\ 1 & 0 & \frac{\partial}{\partial z} & -R_{r p}\end{array}\right] ; \mathcal{Q}=\left[\begin{array}{cccc}K & 0 & 0 & 0 \\ 0 & \frac{1}{\rho} & 0 & 0 \\ 0 & 0 & E I & 0 \\ 0 & 0 & 0 & \frac{1}{I_{\rho}}\end{array}\right]$ and $R_{p}, R_{r p} \in \mathbb{R}$ are nonnegative.

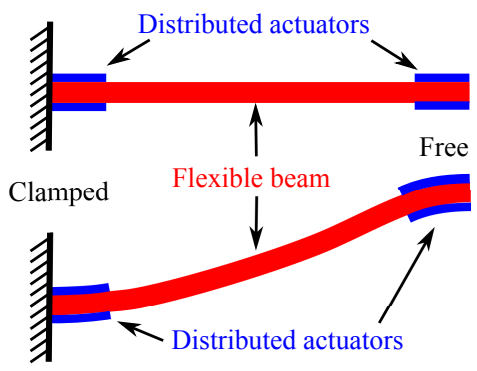

Fig. 1. Example: Timoshenko beam

The energy of the beam is expressed in terms of the energy variables $x_{i}, i \in\{1, \ldots, 4\}$ :

$$
H=\frac{1}{2} \int_{a}^{b}\left(K x_{1}^{2}+\frac{1}{\rho} x_{2}^{2}+E I x_{3}^{2}+\frac{1}{I_{\rho}} x_{4}^{2}\right) d z
$$

where $x_{1}$ is the shear displacement, $x_{2}$ is the transverse momentum distribution, $x_{3}$ is the angular displacement and $x_{4}$ is the angular momentum distribution. The coefficients $\rho$, 
$I_{\rho}, E, I$ and $K$ are the mass per length unit, the rotary moment of inertia of a cross section, the elasticity Young's modulus of elasticity, the moment of inertia of a cross section, and the shear modulus respectively. The state space is defined by $X=L_{2}\left(0,1 ; \mathbb{R}^{4}\right)$. The beam is clamped at the extremity $z=0$, and free at $z=1$, i.e., $\frac{1}{\rho} x_{2}(0, t)=\frac{1}{I_{\rho}} x_{4}(0, t)=0$ $\forall t \geq 0$ and $K x_{1}(1, t)=E I x_{3}(1, t)=0 \forall t \geq 0$, The domain of the operator $\mathcal{M}$ is

$D(\mathcal{M})=\left\{\begin{array}{l|l}x \in H_{1}\left(0,1 ; \mathbb{R}^{n}\right) & \begin{array}{l}x_{2}(0, t)=0 \\ x_{4}(0, t)=0 \\ x_{1}(1, t)=0 \\ x_{3}(1, t)=0\end{array}, \forall t \geq 0\end{array}\right\} \subset X$

with $H_{p}\left(0,1 ; \mathbb{R}^{n}\right)$ the Sobolev space of order $p$. One can check by integration by parts that the domain of the adjoint operator $\mathcal{M}^{*}$ is the same as $\mathcal{M}$.

The operator $\mathcal{Q}$ is self-adjoint and coercive. The parameters $\rho, I_{\rho}, E, I$ and $K$ are considered constant. We consider the beam is actuated by a distributed torque $b_{1}(z) u_{1}(t)$ on the small interval $I_{b_{1}}=[0,0.1]$, with $b_{1}(z)=1$ for $z \in I_{b_{1}}$ and $b_{1}(z)=0$ elsewhere, and also by another distributed torque $b_{2}(z) u_{2}(t)$ on the small interval $I_{b_{2}}=[0.9,1]$, with $b_{2}(z)=1$ for $z \in I_{b_{2}}$ and $b_{2}(z)=0$ elsewhere. As output, we consider the angular velocity mean values over the same intervals $y_{1}=\int_{0}^{1} b_{1}(z) \frac{1}{I_{\rho}} x_{4} d z$ and $y_{2}=\int_{0}^{1} b_{2}(z) \frac{1}{I_{\rho}} x_{4} d z$. As consequence the input operator is:

$$
\mathcal{B}=\left[\begin{array}{cc}
0 & 0 \\
0 & 0 \\
0 & 0 \\
b_{1}(z) & b_{2}(z)
\end{array}\right]
$$

where $\mathcal{B}: \mathbb{C}^{2} \mapsto X$. The output is the conjugated to this input, i.e. $y=\mathcal{B}^{*} \mathcal{Q} x$.

We can verify that the scalar product

$$
\operatorname{Re}\langle\mathcal{M} z, z\rangle_{X} \leqslant 0 \forall z \in D(\mathcal{M})
$$

The operator $\mathcal{M}$ is dissipative, and $\mathcal{M}$ generates a $C_{0}$ semigroup. Furthermore there exists a $0<\lambda_{0} \in \rho(\mathcal{M})$ such that $\operatorname{Ran}\left(\lambda_{0} I-\mathcal{M}\right)=X[14$, Lemma 2.1.11], then $\mathcal{M}$ is $m$-dissipative. It is a generator of a contraction semigroup.

As consequence, the system (10) satisfies all the same conditions as system (1)

\section{PASSIVE LQG CONTROL DESIGN OF INFINITE DIMENSIONAL PHS}

The passive LQG control design has been applied to finite dimensional positive real systems in [15]. In [2] a similar approach equivalent to the Control by Interconnection [6], [16] has been proposed. In this section, we extend this approach to infinite dimensional port Hamiltonian systems.

\section{A. LQG control of infinite dimensional port Hamiltonian systems}

In order to apply the LQG control method to infinite dimensional port Hamiltonian systems we make the following assumption.
Assumption 4. The IDPHS (1) with the m-dissipative operator $\mathcal{M}$ and $(\mathcal{M Q}, \mathcal{B})$ is exponentially stabilizable, i.e., there exists an operator $K \in \mathcal{L}\left(X, \mathbb{C}^{p}\right)$ such that $\mathcal{M Q}-\mathcal{B} K$ generates an exponentially stable semigroup. We also consider that $\left(\mathcal{M Q},-, \mathcal{B}^{*} \mathcal{Q}\right)$ is exponentially detectable, i.e., there exists an operator $F \in \mathcal{L}\left(\mathbb{C}^{p}, X\right)$ such that the operator $\mathcal{M Q}-F \mathcal{B}^{*} \mathcal{Q}$ generates an exponentially stable semigroup.

The LQG control problem of IDPHS (1) is defined as follows:

Problem 5. LQG control problem [14]

Let $\tilde{\boldsymbol{Q}}, \boldsymbol{Q}_{v} \in \mathcal{L}(X)$ be self-adjoint positive definite operators, $\tilde{\boldsymbol{R}}, \boldsymbol{R}_{w} \in \mathcal{L}\left(\mathbb{C}^{p}\right)$ also be self-adjoint strictly positive definite operators and $x \in D(\mathcal{M})$. Then the state feedback gain $K=\tilde{\boldsymbol{R}}^{-1} \mathcal{B}^{*} P_{c}$ with $P_{c}$ the unique positive-definite solution of the operator Riccati equation:

$$
\left(\mathcal{Q} \mathcal{M}^{*} P_{c}+P_{c} \mathcal{M} \mathcal{Q}-P_{c} \mathcal{B} \tilde{\boldsymbol{R}}^{-1} \mathcal{B}^{*} P_{c}+\tilde{\boldsymbol{Q}}\right) x=0
$$

is such that $\mathcal{M Q}-\mathcal{B} \tilde{\boldsymbol{R}}^{-1} \mathcal{B}^{*} P_{c}$ generates an exponentially stable semigroup. The filter gain is $F=P_{f} \mathcal{B R}_{w}^{-1}$ where $P_{f}$ is the unique positive definite solution of

$$
\left(\mathcal{M} \mathcal{Q} P_{f}+P_{f} \mathcal{Q} \mathcal{M}^{*}-P_{f} \mathcal{Q B} \boldsymbol{R}_{w}^{-1} \mathcal{B}^{*} \mathcal{Q} P_{f}+\boldsymbol{Q}_{v}\right) x=0
$$

is such that $\mathcal{M Q}-P_{f} \mathcal{Q B R}{ }_{w}^{-1} \mathcal{B}^{*} \mathcal{Q}$ generates an exponentially stable semigroup.

Thus, the control design problem consists to solve the Riccati equations (16) and (17) in order to minimize the following control cost function:

$$
J_{c o}=\int_{0}^{\infty}\langle x, \tilde{\boldsymbol{Q}} x\rangle_{X}+\langle u, \tilde{\boldsymbol{R}} u\rangle_{\mathbb{C}^{p}} d t
$$

and the estimation error $e(t)=x(t)-x_{c}(t)$ where $x_{c}$ is the estimation of the state (and also the state of the $L Q G$ controller).

The dynamic controller associated with the LQG control Problem 5 can be written as:

$$
\left\{\begin{aligned}
\dot{x}_{c}= & \left(\mathcal{M Q}-\mathcal{B} \tilde{\mathbf{R}}^{-1} \mathcal{B}^{*} P_{c}-P_{f} \mathcal{Q B} \mathbf{R}_{w}^{-1} \mathcal{B}^{*} \mathcal{Q}\right) x_{c} \\
& +P_{f} \mathcal{Q B R} \mathbf{R}_{w}^{-1} u_{c} \\
y_{c}= & \tilde{\mathbf{R}}^{-1} \mathcal{B}^{*} P_{c} x_{c}
\end{aligned}\right.
$$

The closed-loop system with the above LQG controller is stable but not passive in general because the Hamiltonian structure is lost in the closed-loop formulation.

\section{B. LQG formulation of Control by Interconnection}

In order to design a passive LQG controller which can be seen as Control by Interconnection [6], one has to transform the control law (19) into the one of Fig. 2 where the controller has an IDPHS structure. In this perspective, the LQG controller (19) can be rewritten as:

$$
\begin{aligned}
\dot{x}_{c}= & \left(\mathcal{M}-\mathcal{B} \tilde{\mathbf{R}}^{-1} \mathcal{B}^{*} P_{c} \mathcal{Q}^{-1}-P_{f} \mathcal{Q B} \mathbf{R}_{w}^{-1} \mathcal{B}^{*}\right) \mathcal{Q} x_{c} \\
& +P_{f} \mathcal{Q B R} \mathbf{R}_{w}^{-1} u_{c} \\
= & \left(\mathcal{J}-\mathcal{R}_{c}\right) \mathcal{Q} x_{c}+P_{f} \mathcal{Q B} \mathbf{R}_{w}^{-1} u_{c} \\
y_{c}= & \tilde{\mathbf{R}}^{-1} \mathcal{B}^{*} P_{c} \mathcal{Q}^{-1} \mathcal{Q} x_{c}
\end{aligned}
$$




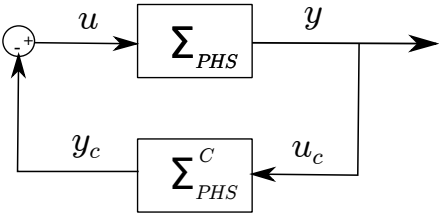

Fig. 2. Control by Interconnection

with $\mathcal{R}_{c}=\mathcal{R}+\mathcal{B} \tilde{\mathbf{R}}^{-1} \mathcal{B}^{*} P_{c} \mathcal{Q}^{-1}+P_{f} \mathcal{Q B} \mathbf{R}_{w}^{-1} \mathcal{B}^{*}$.

In this expression the state operator is decomposed into the product $\left(\mathcal{J}-\mathcal{R}_{c}\right) \mathcal{Q}$ with the energy operator $\mathcal{Q}$ defined in (1). The operator $\mathcal{R}_{c}=\mathcal{R}+\mathcal{B} \tilde{\mathbf{R}}^{-1} \mathcal{B}^{*} P_{c} \mathcal{Q}^{-1}+P_{f} \mathcal{Q B} \mathbf{R}_{w}^{-1} \mathcal{B}^{*}$ is in general neither self-adjoint nor positive. Next we derive the conditions on the LQG control Problem 5 such that the controller (20) has a port Hamiltonian realization.

Theorem 6. Hamiltonian $L Q G$ method

The LQG controller designed using Problem 5 considering

$$
\tilde{\boldsymbol{R}}=\boldsymbol{R}_{w} .
$$

and $\tilde{\boldsymbol{Q}}$ and $\boldsymbol{Q}_{v}$ such that:

$$
\boldsymbol{Q}_{v} z=\mathcal{Q}^{-1}\left(2 \mathcal{Q} \mathcal{J}^{*} P_{c}+2 P_{c} \mathcal{J} \mathcal{Q}+\tilde{\boldsymbol{Q}}\right) \mathcal{Q}^{-1} z,
$$

with $z \in X$, is passive and has a port Hamiltonian realization. Furthermore the operator equations (16) and (17) admit a unique solution, $P_{c}$ and $P_{f}$ respectively. These two solutions are related by:

$$
\mathcal{Q}^{-1} P_{c}=P_{f} \mathcal{Q}
$$

Proof. First, $\mathcal{Q}$ being bounded and coercive and $\tilde{\mathbf{Q}}$ being a positive and self adjoint operator, from (22) and (16) $\mathbf{Q}_{v}$ is bounded. Let's first show that conditions (21) and (22) imply the two solutions $P_{c}$ and $P_{f}$ of the Riccati equations (16) and (17) are related through (23). From (21) and (22), since $\mathcal{Q}$ is invertible, the filter Riccati equation (17) is equivalent to:

$$
\begin{gathered}
\left(\mathcal{Q M} \mathcal{Q} P_{f} \mathcal{Q}+\mathcal{Q} P_{f} \mathcal{Q} \mathcal{M}^{*} \mathcal{Q}-\mathcal{Q} P_{f} \mathcal{Q B} \tilde{\mathbf{R}}^{-1} \mathcal{B}^{*} \mathcal{Q} P_{f} \mathcal{Q}\right. \\
\left.+2 \mathcal{Q} \mathcal{J}^{*} P_{c}+2 P_{c} \mathcal{J} \mathcal{Q}+\tilde{\mathbf{Q}}\right) z=0 .
\end{gathered}
$$

Subtracting the control Riccati equation (16) from (24), one can get after some rearrangements the matching equation :

$$
\begin{aligned}
&\left(P_{c} \mathcal{B} \tilde{\mathbf{R}}^{-1} \mathcal{B}^{*} P_{c}-\mathcal{Q} P_{f} \mathcal{Q} \mathcal{B} \tilde{\mathbf{R}}^{-1} \mathcal{B}^{*} \mathcal{Q} P_{f} \mathcal{Q}+\right. \\
& \mathcal{Q}\left(\mathcal{J}^{*}+\mathcal{R}^{*}\right)\left(P_{c}-\mathcal{Q} P_{f} \mathcal{Q}\right)+ \\
&\left.\left(P_{c}-\mathcal{Q} P_{f} \mathcal{Q}\right)(\mathcal{J}+\mathcal{R}) \mathcal{Q}\right) z=0
\end{aligned}
$$

By using Assumption 4, the IDPHS (1) is exponentially stabilizable and exponentially detectable. As a consequence, from the boundedness of $\tilde{\mathbf{Q}}$ and $\mathbf{Q}_{v}$ both filter and control Riccati equations admit a unique solution. $P_{c}$ and $P_{f}$ being the unique solutions of (16) and (17) respectively, the only solution of (25) is then

$$
P_{c}=\mathcal{Q} P_{f} \mathcal{Q}
$$

which is equivalent to (23). We now show the proposed LQG controller is equivalent to a passive port Hamiltonian system. First by using conditions (21) and (23), the controller output
(20) becomes $y_{c}=\left(\mathbf{R}_{w}^{-1} \mathcal{B}^{*} Q P_{f}\right) \mathcal{Q} x_{c}$, which means that the output mapping of the controller (20) is the adjoint of its input mapping. Second, if we consider conditions (21) and (23), one can check that the operator

$$
\mathcal{R}_{c}=\mathcal{R}+\mathcal{B} \tilde{\mathbf{R}}^{-1} \mathcal{B}^{*} P_{c} \mathcal{Q}^{-1}+\mathcal{Q}^{-1} P_{c} \mathcal{Q B} \tilde{\mathbf{R}}^{-1} \mathcal{B}^{*}
$$

is self-adjoint, i.e., $\mathcal{R}_{c}=\mathcal{R}_{c}^{*}$. Furthermore we recall Assumptions 2 and 4 . First, the domain of the operator $\mathcal{M}$ equals the domain of $\mathcal{M}^{*}$, i.e. $D(\mathcal{M})=D\left(\mathcal{M}^{*}\right)$. Second, the system (1) is exponentially stabilizable and detectable, thus the operator

$$
\begin{aligned}
\mathcal{M}_{c} \mathcal{Q} & =\left(\mathcal{M}-\mathcal{B} \tilde{\mathbf{R}}^{-1} \mathcal{B}^{*} P_{c} \mathcal{Q}^{-1}-\mathcal{Q}^{-1} P_{c} \mathcal{B} \tilde{\mathbf{R}}^{-1} \mathcal{B}^{*}\right) \mathcal{Q} \\
& =\left(\mathcal{J}-\mathcal{R}_{c}\right) \mathcal{Q}
\end{aligned}
$$

generates an exponentially stable semigroup. The operator $\mathcal{Q}$ being bijective and $\mathcal{M}$ m-dissipative, it implies the operator $\mathcal{R}_{c}$ is nonnegative. As a consequence, the LQG controller has a port Hamiltonian realization, then the closed-loop system can be regraded as the interconnection of two infinite dimensional port Hamiltonian systems.

Remark 7. The choice of the control design parameter $\boldsymbol{Q}_{v}$ or $\tilde{\boldsymbol{Q}}$ is free by using Theorem 6, but the two parameters are related by (22). It means that the parameters $\boldsymbol{Q}_{v}, \boldsymbol{R}_{w}$ and $\tilde{\boldsymbol{Q}}$, $\tilde{\boldsymbol{R}}$ depend on each others. If we choose the optimal control problem first, then the co-variance operators $\boldsymbol{Q}_{v}$ and $\boldsymbol{R}_{w}$ are just control design parameters and have no statistic meaning in the filter design problem. On the other hand, if we consider the filter design problem first, then the weighting operators $\tilde{\boldsymbol{Q}}$ and $\tilde{\boldsymbol{R}}$ have no sense in the classical optimal control problem.

The closed-loop system by using Hamiltonian $L Q G$ controller can be regarded as Control by Interconnection of two port Hamiltonian systems. Hence the structure and passivity are conserved in the closed loop system.

In the next section, we discuss the passivity and structure preserving reduction for the closed-loop port Hamiltonian system through the Hamiltonian LQG method.

\section{Reduced Hamiltonian LQG CONTROL Design}

In this section, we use a LQG balanced realization for the IDPHS (1) with respect to the LQG Gramians associated with the Theorem 6 and the relations (23) and (21) $P_{f} P_{c}=$ $P_{f} \mathcal{Q} P_{f} \mathcal{Q}$. The product of these Gramians is different from the identity. Thus, the states of the IDPHS have the different importance for the LQG control design which allows us to reduce the system by state transformation and reduction.

\section{A. Preliminary results}

We first introduce the Hamiltonian LQG Hankel operator associated with IDPHS (1).

Definition 8. Consider two operators $S \in \mathcal{L}\left(X_{s} ; X\right)$ and $L \in \mathcal{L}\left(X_{L} ; X\right)$ with $X_{S}$ and $X_{L}$ are Hilbert spaces such that the Hamiltonian $L Q G$ Gramians satisfy $P_{c}=$ $S S^{*}$ and $P_{f}=L L^{*}$. Then $\mathcal{H}_{L Q G}=S^{*} L \in \mathcal{L}(X)$ is called Hamiltonian LQG Hankel operator of IDPHS (1). 
In order to reduce the system (1), we give a sufficient condition such that the Hamiltonian Gramians $P_{f}, P_{c}$ and the Hamiltonian LQG Hankel operator $\mathcal{H}_{L Q G}$ are nuclear, i.e., the singluar values of the Hamiltonian LQG Hankel operator are the summable and the state space is separable.

Lemma 9. If we chose the weighting operator $\tilde{\boldsymbol{Q}}$ as

$$
\tilde{\boldsymbol{Q}}=\gamma^{2} \mathcal{C}^{*} \mathcal{C}=\gamma^{2} \mathcal{Q} \mathcal{B B}^{*} \mathcal{Q}, \quad \forall \gamma \in \mathbb{R}
$$

with $\mathcal{C}=\mathcal{B}^{*} \mathcal{Q}$. Then $P_{c}$ and $P_{f}$, the two solutions to the operator Riccati equations (16) and (17) of the $L Q G$ control Problem 5 associated with Theorem 6, are nuclear. Furthermore, The Hamiltonian LQG Hankel operator $\mathcal{H}_{L Q G}$ is also nuclear.

Proof. To prove the nuclearity of the solution $P_{c}$ of Riccati equation (16), we consider the Lyapunov equation of the closed loop system:

$$
\begin{gathered}
{[\underbrace{\left(\mathcal{M Q}-\mathcal{B} \tilde{\mathbf{R}}^{-1} \mathcal{B}^{*} P_{c}\right)^{*}}_{\mathcal{M}_{c o}^{*}} L_{o}+L_{o} \underbrace{\left(\mathcal{M Q}-\mathcal{B} \tilde{\mathbf{R}}^{-1} \mathcal{B}^{*} P_{c}\right)}_{\mathcal{M}_{c o}}} \\
\left.+P_{c} \mathcal{B} \tilde{\mathbf{R}}^{-1} \mathcal{B}^{*} P_{c}+\tilde{\mathbf{Q}}\right] z=0
\end{gathered}
$$

with $z \in D(\mathcal{M})$. By developing this Lyapunov equation, we can get:

$$
\begin{gathered}
\left(\mathcal{Q} \mathcal{M}^{*} L_{o}+L_{o} \mathcal{M Q}-P_{c} \mathcal{B} \tilde{\mathbf{R}}^{-1} \mathcal{B}^{*} L_{o}\right. \\
\left.L_{o} \mathcal{B} \tilde{\mathbf{R}}^{-1} \mathcal{B}^{*} P_{c}+P_{c} \mathcal{B} \tilde{\mathbf{R}}^{-1} \mathcal{B}^{*} P_{c}+\tilde{\mathbf{Q}}\right) z=0
\end{gathered}
$$

with $z \in D(\mathcal{M})$. Then $L_{o}=P_{c}$ and (31) is equivalent to the Riccati equation (16).

The closed-loop system is formulated as

$$
\left\{\begin{aligned}
\dot{x} & =\mathcal{M}_{c o} x+\mathcal{B} u \\
y & =\mathcal{C}_{c o} x+\mathcal{D}_{c o} u
\end{aligned}\right.
$$

with $\mathcal{M}_{c o}=\mathcal{M Q}-\mathcal{B} \tilde{\mathbf{R}}^{-1} \mathcal{B}^{*} P_{c}, \mathcal{C}_{c o}=\left[\begin{array}{c}\tilde{\mathbf{Q}}^{1 / 2} \\ \tilde{\mathbf{R}}^{1 / 2} \mathcal{B}^{*} P_{c}\end{array}\right]$ and $\mathcal{B}, \mathcal{D}_{\text {co }}$ are the input and feedthrough operators of this closedloop system. If we can prove $L_{o}$ is nuclear, then $P_{c}=L_{o}$ is also nuclear.

The solution of Lyapunov equation $(30), L_{o}$ is the observability Gramian of the system (32). By using the theorem of [17, Thm 3.1], if the following conditions

1) The operator $\mathcal{M}_{c o}$ is a generator of a exponentially stable $C_{0}$ semigroup;

2) The system (32) has a finite rank output space hold, then $P_{c}=L_{o}$ is nuclear.

From Assumption 4, the operator $\mathcal{M}_{c o}$ is the generator of an exponentially stable $C_{0}$-semigroup, thus the first condition holds. To satisfy the second condition, we can define the weighting operator $\tilde{\mathbf{Q}}=\gamma^{2} \mathcal{Q B B}{ }^{*} \mathcal{Q}$ with $\gamma \in \mathbb{R}$.Then we get $\tilde{\mathbf{Q}}^{1 / 2}=\gamma \mathcal{B}^{*} \mathcal{Q} \in \mathcal{L}\left(X, \mathbb{C}^{p}\right)$ and $\tilde{\mathbf{R}}^{1 / 2} \mathcal{B}^{*} P_{c} \in \mathcal{L}\left(X, \mathbb{C}^{p}\right)$. Hence the output operator of system (32)

$$
\mathcal{C}_{c o}=\left[\begin{array}{c}
\tilde{\mathbf{Q}}^{1 / 2} \\
\tilde{\mathbf{R}}^{1 / 2} \mathcal{B}^{*} P_{c}
\end{array}\right] \in \mathcal{L}\left(X, \mathbb{C}^{2 p}\right)
$$

maps from the state space $X$ to a finite rank space $\mathbb{C}^{2 p}$, i.e., the system (32) has a finite rank output space, the second condition holds too. As consequence the LQG Gramian $P_{c}$ is nuclear. Similarly we can prove that $P_{f}=\mathcal{Q} P_{c} \mathcal{Q}$ and Hamiltonian LQG Hankel operator $\mathcal{H}_{L Q G}$ are also nuclear .

Following Lemma 9, the Hamiltonian LQG Hankel operator $\mathcal{H}_{L Q G}$ is nuclear. We can then define the balanced realization with respect to the Hamiltonian LQG Gramians $P_{f}$ and $P_{c}$ of the LQG control Problem 5 associated with Theorem 6.

Definition 10. The IDPHS is called Hamiltonian LQG balanced if $X=\ell_{2}$ and there exists positive and non-increasing sequence $\left(\sigma_{n}\right)_{n \in \mathbb{N}}$ such that the Hamiltonian LQG Gramians $P_{f}$ and $P_{c}$ are both equal to the diagonal operator:

$$
P_{f}=P_{c}=\Sigma=\operatorname{diag}\left(\sigma_{k}\right)_{k \in \mathbb{N}} \in \mathcal{L}\left(\ell_{2}\right) .
$$

\section{B. Hamiltonian LQG balanced realization of IDPHS}

Following the Lemma 9, the Hamiltonian LQG Hankel operator $\mathcal{H}_{L Q G}=S^{*} L \in \mathcal{L}(X)$ is nuclear with $S, L \in \mathcal{L}(X)$ and admits a singular value decomposition $S^{*} L=V \Sigma U^{*}$ where $\Sigma=\operatorname{diag}\left(\sigma_{k}\right)_{k \in \mathbb{N}} \in \mathcal{L}\left(\ell_{2}\right)$ with the positive sequence of Hamiltonian LQG Hankel singular values $\left(\sigma_{k}\right)$. The operators $V, U \in \mathcal{L}\left(\ell_{2} ; X\right)$ are isometrics onto their ranges, i.e., $V^{*} V=I, U^{*} U=I$.

Theorem 11. Suppose that the IDPHS (1) and the transformation operators $T$ and $T^{+}$defined in (36), then a balanced realization from (1) is given as

$$
\left\{\begin{aligned}
\dot{x}_{b}(t) & =M_{b} Q_{b} x_{b}(t)+B_{b} u(t) \\
y(t) & =B_{b}^{*} Q_{b} x_{b}(t)
\end{aligned}\right.
$$

with $M_{b}=T \mathcal{M} T^{*}, Q_{b}=T^{+*} \mathcal{Q} T^{+}, B_{b}=T \mathcal{B}$ and

$$
\begin{aligned}
& T:=\Sigma^{-1 / 2} V^{*} S^{*} \subset X \mapsto \ell_{2} ; \\
& T^{+}:=L U \Sigma^{-1 / 2} \subset \ell_{2} \mapsto X .
\end{aligned}
$$

The state space of the balanced IDPHS is $x_{b} \in \ell_{2}$.

Proof. The two solutions of Riccati equations have the following decomposition $P_{c}=S S^{*}$ and $P_{f}=L L^{*}$. We can compute $T P_{f} T^{*}=\Sigma^{-1 / 2} V^{*} S^{*} L L^{*} S V \Sigma^{-1 / 2}$, with the SVD of Hamiltonian LQG Hankel operator $S^{*} L=V \Sigma U^{*}$, then $T P_{f} T^{*}=\Sigma$. We can compute $T^{+} P_{c} T^{+*}=\Sigma$ in the same way. As a consequence, we get the Hamiltonian LQG Gramians of the system (35) as follows:

$$
T P_{f} T^{*}=T^{+} P_{c} T^{+*}=\Sigma .
$$

The system (35) is the Hamiltonian LQG balanced realization of the IDPHS (1).

This balanced realization is defined on an $\ell_{2}$ space, and the state variables are separated and arranged in decreasing order according to their importance in the closed-loop system defined from the Hamiltonian LQG singular values. In other words, the state variables associated with large singular values are more important for the Hamiltonian LQG control design than the other ones. Hence from the closed-loop point of view, this balanced realization gives us the good choice of state space to reduce the IDPHS. This reduction method is derived in the next sub-section.

Remark 12. To get the $L Q G$ balanced realization, an important step is to solve the operator Riccati equations, which is 
almost impossible to solve in the infinite dimensional case. The semi-discretization of the system (1) is needed to compute operator Riccati equations.

\section{Reduction and reduced order controller design of IDPHS}

In order to preserve the passivity and the Hamiltonian structure of the system after the reduction, a direct truncation cannot be used and we propose to adapt the Petrov-Galerkin projection method [7]. In what follows $n$ is the order of reduction we choose. Consequently the reduced LQG Grammians reduce to:

$$
\Sigma_{1}=\operatorname{diag}\left(\sigma_{k}\right)_{k=1, \cdots, n}
$$

1) Petrov-Galerkin projection method: In Petrov-Galerkin projection method the state variables are decomposed in $x(t)=x_{n}(t)+x_{r}(t)$ with $x(t) \in X, x_{n}(t) \in V$ and $x_{r}(t) \in W^{\perp}$, where $V=\operatorname{span}\left\{v_{1}, \cdots, v_{n}\right\}$ and $W=$ $\operatorname{span}\left\{w_{1}, \cdots, w_{n}\right\}$ with $v_{i}$ in the state operator domain $\left(v_{i} \in\right.$ $D(\mathcal{M Q})$ in system (1)) and $w_{i} \in X$ the n-dimensional subspaces of $X$, and $W^{\perp}$ the orthogonal complement of $W$. This decomposition exists and is unique if $V \cap W^{\perp}=\{0\}$. The linear operators $\mathcal{V}: \mathbb{C}^{n} \mapsto X$ and $\mathcal{W}: X \mapsto \mathbb{C}^{n}$ defined by:

$$
\mathcal{V} \alpha=\sum_{i=1}^{n} v_{i} \alpha_{i} \quad \mathcal{W} h=\left[\begin{array}{c}
\left\langle h, w_{1}\right\rangle_{X} \\
\vdots \\
\left\langle h, w_{n}\right\rangle_{X}
\end{array}\right]
$$

for all $\alpha \in \mathbb{C}^{n}, h \in X$ are such that $V \cap W^{\perp}=\{0\}$. This property can be easily verified by using $\operatorname{det}(\mathcal{W V}) \neq 0$ where $\mathcal{W V} \in \mathbb{C}^{n \times n}$. Thus one can use the internal direct sum decomposition $X=V \oplus W^{\perp}$ if the choices of $W$ and $V$ are such that $\operatorname{det}(\mathcal{W V}) \neq 0$. In order to determine a finitedimensional model that describes the dynamics of $x_{n}$ it is advantageous to introduce the projection $\mathcal{P}: X \mapsto V$ of $X$ onto $V$ along $W^{\perp}$, yielding the relation $x_{n}(t)=\mathcal{P} X(t)$. This projection can be expressed as $\mathcal{P}=\mathcal{V}(\mathcal{W V})^{-1} \mathcal{W}$ and satisfies $\mathcal{P}=\mathcal{P}^{2}$. Its range and null space satisfy $\operatorname{Ran} \mathcal{P}=V$, $\operatorname{Ker} \mathcal{P}=W^{\perp}$.

2) Passivity and structure preserving approach: To preserve the passivity of the port Hamiltonian system (1) by using the Petrov-Galerkin projection method, a special choice of operator $\mathcal{V}$ and $\mathcal{W}$ is given in [7]. In this method, the authors did not give the choice of vectors $v_{i}$.

Inspired by this method we shall introduce a choice of vectors $v_{i}$ which define the projection operator $\mathcal{V}$ and $\mathcal{W}$ to preserve the passivity and Hamiltonian structure through the balanced reduction of system (35):

Theorem 13. Define $\mathcal{V}: \mathbb{C}^{n} \mapsto \ell_{2}$ by

$$
\mathcal{V} z=\sum_{i=1}^{n} v_{i} z_{i} \quad \forall z_{i} \in \mathbb{C}^{n}, \quad i \in \mathbb{N}
$$

with $v_{i}=\left(\delta_{i, 1}, \delta_{i, 2}, \cdots\right) \in \ell_{2}$ is the canonical unit vector. Consider the special choice $\mathcal{W}=\mathcal{V}^{*} Q_{b}$. Then a structure preserving approximation of the infinite-dimensional DPHS is a linear DPHS:

$$
\left\{\begin{aligned}
\dot{x}_{n} & =M_{n} Q_{n} x_{n}+B_{n} u \\
y & =B_{n}^{*} Q_{n} x_{n}
\end{aligned}\right.
$$

with $M_{n}=\mathcal{V}^{*} Q_{b} M_{b} Q_{b} \mathcal{V}, Q_{n}=\left(\mathcal{V}^{*} Q_{b} \mathcal{V}\right)^{-1}$ and $B_{n}=$ $\mathcal{V}^{*} Q_{b} B_{b}$

Proof. First we can show that $\mathcal{P}=\mathcal{V}(\mathcal{W V})^{-1} \mathcal{W}=\mathcal{P}^{2}$ is a projection. Then we choose $x_{b} \approx \mathcal{V} z_{n}$ and premultiple (35) the operator $\mathcal{W}=\mathcal{V}^{*} Q_{b}$. The finite-dimensional approximation becomes

$$
\left\{\begin{aligned}
\mathcal{V}^{*} Q_{b} \mathcal{V} \dot{z}_{n} & =\mathcal{V}^{*} Q_{b} M_{b} Q_{b} \mathcal{V} z_{n}+\mathcal{V}^{*} Q_{b} B_{b} u(t) \\
y(t) & =B_{b}^{*} Q_{b} \mathcal{V} z_{n}
\end{aligned}\right.
$$

We choose $x_{n}=\mathcal{V}^{*} Q_{b} \mathcal{V} z_{n}$. The matrix $M_{n}$ can be separated in two parts, one part is skew symmetric and the other part is symmetric positive definite since $M_{n}+M_{n}^{*} \leq 0$ and

$$
J_{n}=\frac{1}{2}\left(M_{n}-M_{n}^{*}\right) \text { and } R_{n}=-\frac{1}{2}\left(M_{n}+M_{n}^{*}\right)
$$

By using the finite dimensional PHS (41) and the LQG control Problem 5 associated with Theorem 6, we design a finite dimensional controller in order to stabilize the IDSHP (8) following Algorithm 1. Le final controller of order $n$ is on the form

$$
\left\{\begin{array}{l}
\dot{x}_{c n}=\left(J_{c n}-R_{c n}\right) Q_{n} x_{c n}+B_{c n} u_{c} \\
y_{c n}=B_{c n}^{T} Q_{n} x_{c n}
\end{array}\right.
$$

where

$$
\begin{aligned}
J_{c n}-R_{c n} & =M_{n}-B_{n} \tilde{R}^{-1} B_{n}^{T} \Sigma_{1} Q_{n}^{-1}-\Sigma_{1} Q_{n} B_{n} R^{-1} B_{n}^{T} \\
B_{c n} & =\Sigma_{1} Q_{n} B_{n} R^{-1}
\end{aligned}
$$

and is strictly positive real [15, Definition 2.42] which can be verified by the Lefschetz-Kalman-Yakubovich Lemma [15, Lemma 3.11].

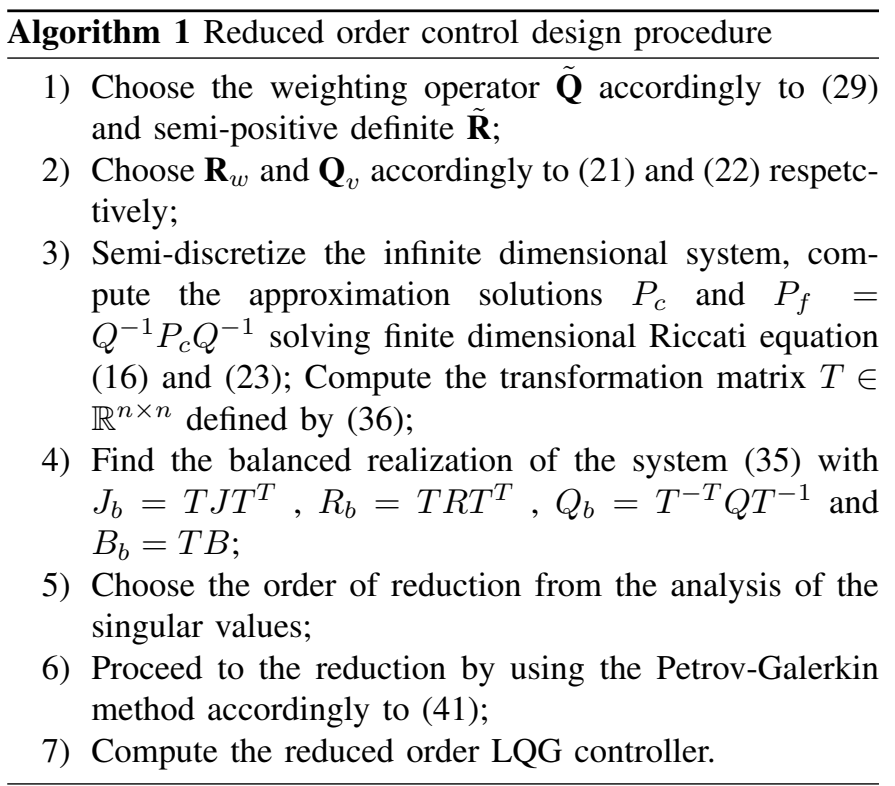

\section{Closed-loop stability}

In this subsection we consider the closed-loop stability of the infinite dimensional system connected to the finite dimensional LQG controller (43). We first consider the existence of solution in Theorem 14. 
Theorem 14. Let the state of the open loop system of (8) satisfy $\frac{1}{2} \frac{d}{d t}\|x(t)\|_{\mathcal{Q}}^{2}=u(t)^{T} y(t)$ as described in (6) and let the controller (43) being strictly positive real. Then the closedloop system is defined by

$$
\dot{w}=\mathcal{J}_{c l} w(t), w(0) \in \tilde{X}
$$

where $\tilde{X}=X \times \mathbb{R}^{m}$ is the state space of the closed-loop system, $w=\left[\begin{array}{c}x \\ x_{c n}\end{array}\right] \in \tilde{X}$ and $\mathcal{J}_{c l}: \tilde{X} \rightarrow \tilde{X}$ is a linear operator defined by

$$
\mathcal{J}_{c l} w=\left[\begin{array}{cc}
(\mathcal{J}-\mathcal{R}) \mathcal{Q} & -\mathcal{B} B_{c n}^{T} Q_{c n} \\
B_{c n} \mathcal{B}^{*} \mathcal{Q} & \left(J_{c n}-R_{c n}\right) Q_{c n}
\end{array}\right]\left[\begin{array}{c}
x \\
x_{c n}
\end{array}\right]
$$

with

$$
D\left(\mathcal{J}_{c l}\right)=H_{N}\left(0,1 ; \mathbb{R}^{n}\right) \times \mathbb{R}^{m}
$$

Furthermore the operator $\mathcal{A}_{c}$ defined by $\mathcal{A}_{c} w=\mathcal{J}_{c l} w$ with

$$
D\left(\mathcal{A}_{c}\right)=D(\mathcal{M}) \times \mathbb{R}^{m}
$$

generates a contraction semigroup on $\tilde{X}$.

Proof. The closed-loop operator is a consequence of the power preserving interconnection of (8) with (43). Due to the maximal dissipativity of $(\mathcal{J}-\mathcal{R}) \mathcal{Q}$, the strict positive realness of (43), one can show that $\mathcal{A}_{c}$ and $\mathcal{A}_{c}^{*}$ are maximal dissipative on $\mathcal{D}\left(\mathcal{A}_{c}\right)$ and $\mathcal{D}\left(\mathcal{A}_{c}^{*}\right)$ respectively. Then from Lümer-Phillips theorem [18] $\mathcal{A}_{c}$ generates a contraction semigroup.

Theorem 15. The controller (43) being strictly positive real the closed-loop system (44) is globally asymptotically stable. That is for any $w(0) \in \tilde{X}$, the unique solution of (44) asymptotically approach to zero, i.e., $\lim _{t \rightarrow 0}\|w(t)\|_{\tilde{X}}=0$

Proof. The proof is quite similar to the proof of Theorem 5.9 in [19] where boundary control is considered. The operator $(\mathcal{J}-\mathcal{R}) \mathcal{Q}$ with domain $D(\mathcal{M})$ has compact resolvant by Theorem 2.8 of [19]. By applying Theorem 8.1-3 of [20] we can show that $\left(\lambda I-\mathcal{A}_{c}\right)^{-1}$ is compact for $\lambda=1$, which implies that $\left(\lambda I-\mathcal{A}_{c}\right)^{-1}$ is compact for all $\lambda \in \rho\left(\mathcal{A}_{c}\right)$ by applying Theorem 6.29 of [21]. Asymptotic stability is shown using the closed-loop energy function as Lyapunov function and Lasalle's invariance principe as shown in [19, Theorem 5.10]. From $\frac{1}{2} \frac{d}{d t}\|w(t)\|_{\tilde{\mathcal{Q}}}^{2} \leq-\langle x, \mathcal{R} x\rangle_{X}-x_{c n}^{T} R_{c n} x_{c n}$, the equilibrium profile satisfies $y_{c n}=u_{c n}=0$ and from Assumption 4 it reduces to the only null equilibrium state.

\section{Simulation Results}

In this section, we illustrate the proposed method on the clamped-free Timoshenko beam model of Example 3. Since this model satisfies the same conditions as system 1, one can design the reduced order controller by using the Algorithm 1. In what follows, we illustrate the numerical properties of such control. Differently from the results proposed in [22] the controller we propose is passive and guarantees the closedloop stability when applied to the infinite dimensional system.

For a sake of simplicity all the parameters of the Timoshenko beam have been selected equal to one for the simulations. The damping parameters $R_{p}$ and $R_{p r}$ have been chosen equal to 0.01 in order to show the beam is weakly damped. As mentioned in Remark 12, in order to solve the operator Riccati equation and for the reduced controller design, we use the mixed-finite elements method [23] to discretize the Timoshenko beam model first. The advantage of this discretization method is that the passivity and Hamiltonian structure of the original infinite dimensional system are preserved. In Assumption 4, we assume that the original system is exponentially stabilizable/detectable, then its stabilizability and detectability can be also preserved in its finite dimensional approximation using mixed-finite elements method. Thus, following the results of [24], [5], the solutions of the finite dimensional Riccati equations raising from the mixed-finite elements approximation of the original infinite dimensional system converge to the solution of the operator Riccati equation. For the numerical illustrations and the reduced order controller design, the spatial space of beam has been divided in 10 infinitesimal subsections, hence the system has 40 state variables.

First, we use Theorem 6 to design the controller and reduce the system by taking the weighting operator $\tilde{\mathbf{Q}}=\mathcal{Q B B}^{*} \mathcal{Q}$. Fig. 3 shows the LQG singular values. One can observe that the first 6 singular values are larger than the others. That means the first 6 states of the balanced system play the most important role in the closed-loop system. As a consequence, we reduce the balanced system to order 6 using Theorem 13, then we design a reduced order controller on this reduced system.

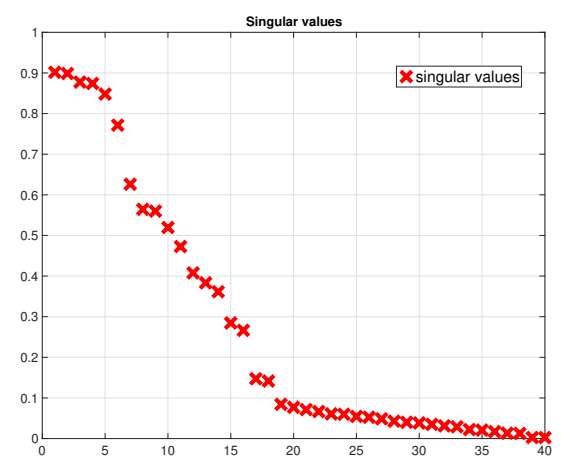

Fig. 3. LQG singular values

In Fig. 4, we compare the two closed-loop systems by interconnecting the full order controller $\left(x_{c} \in \mathbb{R}^{40}\right.$, the black dashed curve) and reduced order controller $\left(x_{c r} \in \mathbb{R}^{6}\right.$, the red solid curve) with the open loop system (The blue dotted curve). It is important to notice that we use the obtained controllers to stabilize a high order system (200 infinitesimal subsections, $x \in \mathbb{R}^{800}$ ) which can be regarded as the infinite dimensional system. The control objective is to stabilize the beam around a desired equilibrium position corresponding to a normalized moment step applied at the tip of the beam (purple dotted line).

One can observe that the closed-loop systems with full order and reduced order controllers have almost the same performances but have a little gap (the difference is less than 0.1 ) in the overshoot peaks as shown in Fig. 4 that is due to the fact that the reduced controller has only an order 6 . In Fig. 4 we also compare the proposed reduced order LQG 
controller with another classical reduced order controller. This controller is designed based on the classical LQG reduction method with the same reduced order, i.e., $x_{c r} \in \mathbb{R}^{6}$ (cyan line). In this LQG control design, we take the same optimal control weighting operator as the passive one i.e., $\tilde{\mathbf{Q}}=\mathcal{Q B B}^{*} \mathcal{Q}$, but the weighting operator of the filter problem is chosen as $\mathbf{Q}_{c}=\mathcal{B B}^{*}$ instead of computing by Theorem 6. One can observe that the closed-loop response diverges in the finite time (spillover effect) since the Hamiltonian structure and passivity of the system are lost during the reduction and control design procedure by using this method.

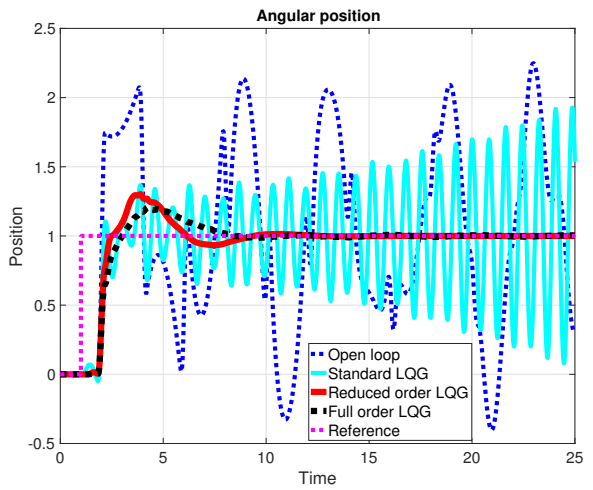

Fig. 4. Comparison of the closed-loop performances obtained by using the different controllers.

\section{CONCLUSION}

In this paper we first proposed a specific LQG control design for IDPHS that guarantees the resulting dynamic controller is passive and can be formulated a passive port Hamiltonian system. In this case the control is equivalent to Control by Interconnection. It has been done through a specific choice of the weighting operators used in the optimal control and filter design problems.

However, the passive LQG controller obtained by using this Hamiltonian LQG method is still infinite dimensional as the system itself. Hence we suggested a reduce-then-design scheme to get a finite dimensional controller for the IDPHS using the Petrov-Galerkin projection method. The reduced order controller is still passive and we proved the closed-loop stability when applied to the infinite dimensional system. In the last part, the proposed approach is illustrated on the control of a Timoshenko beam.

The ongoing work of this research is the following: first, the main difficulty of the proposed method lies in the resolution of the operator Riccati equations. It has been done by using a finite approximation of the considered operators but the effect of such discretization remains to be studied. Second, as in the finite dimensional case, the estimation of the error due to the approximation has to be characterized or at least bounded. At last, in this paper only input control within the domain with bounded operators has been considered. All the proposed results still have to be generalized to boundary control, with all the theoretical difficulties associated with the use of unbounded operators. Those three problems shall be investigated in the future research.

\section{REFERENCES}

[1] M. J. Balas, "Active control of flexible systems," Journal of Optimization Theory and Applications, vol. 25, pp. 415-436, Jul 1978.

[2] Y. Wu, B. Hamroun, Y. L. Gorrec, and B. Maschke, "Reduced order LQG control design for Port Hamiltonian systems," Automatica, vol. 95, pp. $86-92,2018$.

[3] K. A. E. Camp and B. B. King, "A Comparison of Balanced Truncation Techniques for Reduced Order Controllers," Proc. of Math. Theory of Networks and Systems, Notre, 2002.

[4] B.B.King, N. Hovakimyan, K. Evans, and M.Buhl, "Reduced Order Controllers for Distributed Parameter Systems: LQG balanced Truncation and an Adaptive Approach," Mathematical and Computer Modelling, vol. 43, no. 9-10, pp. 1136 - 1149, 2006.

[5] R. F. Curtain, "Model reduction for control design for distributed parameter systems," Research Directions in Distributed Parameter Systems, pp. 95-121, 2003.

[6] R. Ortega, A. van der Schaft, I. Mareels, and B. Maschke, "Putting energy back in control," IEEE Control Systems Magazine, vol. 21, pp. 18- 32, April 2001.

[7] C. Harkort and D. Deutscher, "Stability and Passivity Preserving PetrovGalerkin Approximation of Linear Infinite-Dimensional Systems," $\mathrm{Au}$ tomatica, vol. 48, no. 7, pp. 1347-1352, 2012.

[8] Z. Liu and S. Zheng, Semigroups Associated with Dissipative Systems. Chapman \& Hall/CRC Research Notes in Mathematics Series, Taylor \& Francis, 1999.

[9] A. van Der Schaft, $L_{2}$-gain and Passivity Techniques in Nonlinear Control. Communications and Control Engineering Series, SpringerVerlag, 2000

[10] A. van der Schaft and B. Maschke, "Hamiltonian Formulation of Distributed Parameter Systems with Boundary Energy Flow," Journal of Geometry and Physics, vol. 42, pp. 166-194, 2002.

[11] Y. Le Gorrec, H. Zwart, and B. Maschke, "Dirac Structures and Boundary Control Systems Associated with Skew-symmetric Differential Operators," SIAM Journal on Control and Optimization, vol. 44, pp. 1864-1892, 2005

[12] B. Jacob and H. Zwart, Linear Port-Hamiltonian Systems on Infinitedimensional Spaces. Operator Theory, Springer Basel, 2012.

[13] A. Macchelli and C. Melchiorri, "Modeling and Control of the Timoshenko Beam. The Distributed Port Hamiltonian Approach," SIAM Journal on Control and Optimization, vol. 43, no. 2, pp. 743-767, 2004.

[14] R. Curtain and H. Zwart, An introduction to Infinite-Dimensional Linear System Theory. Springer-Verlag, i ed., 1995. ISBN 0-387-94475-3.

[15] B. Brogliato, R. Lozano, B. Maschke, and O. Egeland, Dissipative Systems Analysis And Control: Theory And Applications. Communications and Control Engineering, Springer-Verlag London, 2007. Second edition.

[16] R. Ortega, A. van der Schaft, F. Castanos, and A. Astolfi, "Control by interconnection and standard passivity-based control of port-hamiltonian systems," IEEE Trans. on Automatic Control, vol. 53, no. 11, 2008.

[17] R. F. Curtain and A. J. Sasane, "Compactness and nuclearity of the hankel operator and internal stability of infinite-dimensional state linear systems," International Journal of Control, vol. 74, no. 12, pp. 12601270, 2001.

[18] A. Pazy, Semigroups of Linear Operators and Applications to Partial Differential Equations. 1983.

[19] J. Villegas, A Port-Hamiltonian Approach to Distributed Parameter Systems. PhD thesis, University of Twente, Enschede, The Netherlands, May 2007.

[20] Introdutory functional analysis withapplications. John Wiley and Sons, New York, 1989.

[21] T. Kato, Perturbation theory for Linear Operators (2nd Edition). Springer-Verlag New York, Inc., 1995.

[22] P. Braun, E. Hernández, and D. Kalise, "Reduced-order lqg control of a timoshenko beam model," Bulletin of the Brazilian Mathematical Society, New Series, vol. 47, pp. 143-155, Mar 2016.

[23] G. Golo, V. Talasila, A. van der Schaft, and B. Maschke, "Hamiltonian Discretization of Boundary Control Systems," Automatica, vol. 40, pp. 757-771, 2004.

[24] K. Morris, "Convergence of controllers designed using state-space techniques," Automatic Control, IEEE Transactions on, vol. 39, pp. 2100 2104, Oct 1994. 